\title{
Exploring Modular Glycolipids Involved in Nematode Chemical Communication
}

\author{
Stephan H. von Reuss*
}

\begin{abstract}
Chemical communication in nematodes has been known for over half a century, but the underlying molecular basis remained largely elusive. Recent advances in analytical techniques facilitated the characterization of a modular glycolipid library based on the dideoxysugar L-ascarylose, which modulates behavior and development in the model organism C. elegans. Ascaroside signaling is highly conserved in nematodes and represents a key factor in nematode chemical ecology. Ascaroside biosynthesis depends on the co-option of the peroxisomal $\beta$-oxidation cycle and in addition integrates a large diversity of additional building blocks derived from various primary metabolic pathways to give rise to species-specific modular assemblies, thus, transcending the concept of strictly segregated primary versus secondary metabolism.
\end{abstract}

Keywords: Ascarosides · Chemical ecology · Glycolipids · Modular metabolites · Nematodes

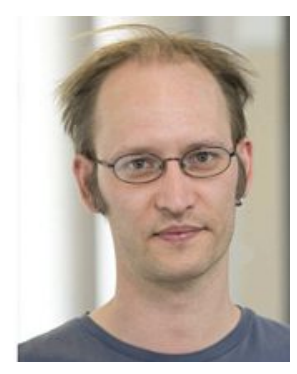

Stephan H. von Reuss (born 1975 in Germany) studied Chemistry at the University of Hamburg. After receiving his MSc (2004) and PhD (2009) for research on secondary metabolites from plants and microorganisms, he joined Cornell University (Ithaca, USA) for postdoctoral studies and started to work with small molecule signals from the model organism Caenorhabditis elegans. In 2012, he returned to Germany to establish his own research group focusing on chemical communication in nematodes at the Max Planck Institute for Chemical Ecology in Jena. In 2016, he was appointed to full professor (professeur ordinaire) at the Institute of Chemistry of the University of

${ }^{*}$ Correspondence: Prof. S. H. von Reuss Laboratory for Bioanalytical Chemistry University of Neuchâtel

Avenue de Bellevaux 51, Neuchâtel, Switzerland

E-mail: stephan.vonreuss@unine.ch
Neuchâtel, where he leads the Laboratory for Bioanalytical Chemistry and serves as director of the Neuchâtel Platform of Analytical Chemistry (NPAC). His major research interests include the development of analytical techniques to decipher the ecology and evolution of secondary metabolism and chemical signaling in nematodes.

\section{Chemical Communication in Nematodes}

Chemical communication in nematodes (roundworms), arguably the most abundant group of animals on earth, has been known for more than half a century, ${ }^{[1]}$ and documented in a multitude of species, irrespective of their various feeding habits. However, despite considerable efforts, molecular structures of nematode-derived small molecule signals remained elusive for several decades. ${ }^{[2]}$ In this mini-review I would like to focus on the development and application of novel analytical techniques that facilitate the discovery and characterization of the ascarosides, a complex modular glycolipid library, which constitutes the structural basis of chemical communication in nematodes and represents a key regulator in nematode chemical ecology. ${ }^{[3]}$

Exploring nematode chemical ecology and secondary metabolism is highly desirable considering that nematodes are ubiquitous in most ecosystems and considered to be of fundamental importance in many belowground interactions. ${ }^{[4]}$ Free-living and parasitic nematodes occupy habitats teeming with microorganisms that are known to produce highly potent bioactive compounds. ${ }^{[5]}$ Consequently, we anticipate sophisticated detoxification mechanisms, the characterization of which might increase our understanding of nematode resistance and facilitate the development of effective antihelminthics and nematicides to control nematode pests that represent a great burden on human health, animal farming, and agriculture. For example, plant parasitic nematodes are responsible for estimated losses equaling $12.3 \%$ of global food production worth US\$ 157 billion per annum. ${ }^{[6]}$ Soil-transmitted gastrointestinal nematodes affect 3.5 billion patients worldwide of which 450 million are seriously ill. ${ }^{[7]}$ Apart from the need to better understand and control parasitic nematodes, the free-living bacterivorous Caenorhabditis elegans constitutes an extremely successful model organism in biology and medicine introduced by Sydney Brenner in $1963,{ }^{[8]}$ which served in several Nobel-prize winning research projects focusing on apoptosis, ${ }^{[9]}$ systematic gene cloning, ${ }^{[10]}$ first complete genome sequencing of a multicellular organism, ${ }^{[11]}$ RNA interference (RNAi), ${ }^{[12]}$ and green fluorescence protein (GFP). ${ }^{[13]}$ While $C$. elegans undoubtedly represents one of the best understood animals today, our knowledge regarding its chemical ecology and secondary metabolism is surprisingly limited.[14] Recent research has demonstrated that $C$. elegans and (possibly all) other nematodes generate a large diversity of highly complex secondary metabolites by the combinatorial assembly of building blocks derived from various primary metabolic pathways (Fig. 1), which represents an unprecedented mechanism to generate structural diversity covering a vast chemical space. Among these modular assemblies a class of glycolipids called ascarosides has been shown to represent key modulators in nematode chemical ecology. ${ }^{[3]}$ 


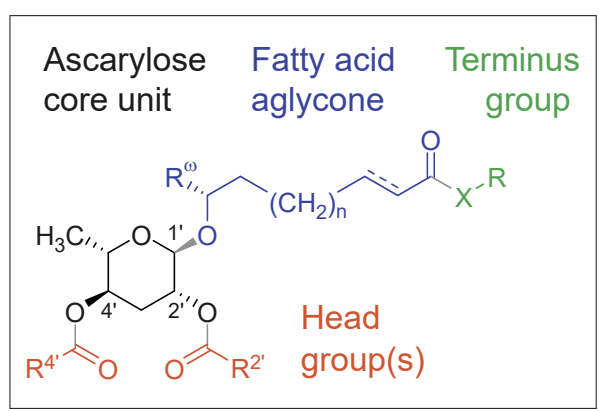

Fig. 1. Modular ascaroside structure originating from the combinatorial assembly of building blocks derived from various primary metabolic pathways.

\section{The Discovery of Ascaroside Signals in C. elegans}

In 1982 Golden and Riddle reported that cultures of the model organism $C$. elegans accumulate an unidentified factor that induces the formation of 'Dauer' larva (from the German word 'Dauer' for enduring), an alternative non-feeding developmental stage optimized for long-term survival.[15] Production of this worm-derived signal depends on the daf22 gene, ${ }^{[16]}$ later shown to represent a 3-ketoacyl-S-CoA thiolase implicated in $\beta$-oxidation of fatty acids, but the molecular structures remained unknown for another two decades. In a seminal work, the Paik group employed bioactivity-guided fractionation to isolate a dauer inducing principle from the $C$. elegans exometabolome that was initially called daumone[17] and subsequently termed ascr\#1 or asc-C7 $(\mathbf{1}, \mathrm{n}=2){ }^{[18]}$ Its $\alpha$-glycosidic structure was shown to be composed of a 3,6-dideoxyL-mannose unit called L-ascarylose, linked to the $(\omega-1)$ position of a heptanoic acid side chain as the aglycone. ${ }^{[17]}$ Additional dauer-inducing ascarosides were subsequently isolated using activity guided fractionation, including the significantly more potent asc-C6-MK $(\mathbf{6}, \mathrm{n}=2$; ascr\#2; daumone\#2) and asc- $\Delta C 9(2, n=4$; ascr\#3; daumone\#3), ${ }^{[19]}$ as well as the $(\omega)$-linked asc- $\omega \mathrm{C} 3$ (8, ascr\#5). ${ }^{[20]}$ Furthermore, it was shown that synergistic blends of these components also modulate nematode behavior in concentrations significantly smaller than those required for dauer induction. ${ }^{[21]}$ Considering the fact that bioactivity guided fractionation is unsuitable for the isolation of synergistically active components, untargeted comparative metabolomics were employed to pursue the identification of additional compounds required in order to reconstitute the full activity of the crude pheromone extract. ${ }^{[22]}$ Differential analysis of 2D NMR spectra (DANS) based on high resolution $d q f$-COSY spectra of the (active) wild type and the (inactive) daf-22 mutant metabolome revealed three additional ascarosides, ascC6-OH (7, ascr\#6), asc- $\Delta \mathrm{C} 7(2, \mathrm{n}=2$; ascr\#7), and asc- $\triangle$ C7-PABA (20, ascr\#8), the first modular ascaroside component, which functions as a potent male attractant in synergism with asc- $\Delta \mathrm{C} 9(\mathbf{2}, \mathrm{n}=4)$ and asc-C6-MK (6). ${ }^{[22]}$ In addition, DANS revealed a group of modular ascaroside derivatives such as $\mathbf{1 3}$ and $\mathbf{1 4}$ that carry an indole-3-carboxylate moiety attached to the 4 '-position of the ascarylose unit, which serve as extremely potent hermaphrodite attractants and aggregation pheromones in femtomolar amounts. [23]

\section{Targeted Mass Spectrometric Screens for Ascaroside Profiling}

Because nematode-derived ascarosides are commonly produced as homologous series that are excreted in minute amounts into a complex background matrix consisting of primary and secondary metabolites, highly sensitive mass spectrometric techniques are indispensable for comprehensive ascaroside analysis. Targeted MS screens that take advantage of specific fragment ions in order to mark chromatographic signals corresponding to potential ascaroside structures, while masking the large majority of background signals, facilitate the detection of (most) known as well as many yet unidentified ascarosides. Targeted screens thus facilitate the comprehensive analysis of ascaroside components, including their derivatives and biosynthetic precursors, irrespective of their potential biological activity or the availability of differential samples. Based on the observation that deprotonated ascaroside anions $[\mathrm{M}-\mathrm{H}]^{-}$ produced during electrospray ionization in negative mode (ESI- $(-)$ ) consistently form a fragment ion at $m / z, 73.1\left[\mathrm{C}_{3} \mathrm{H}_{5} \mathrm{O}_{2}\right]^{-}$upon collision induced dissociation (CID) (Fig. 2A), we have developed a HPLC-ESI-MS/ MS precursor ion screen for $m / z, 73.1$ that facilitates the detection of known as well as previously unidentified ascarosides. ${ }^{[24]}$ Similarly, protonated ascaroside cations $[\mathrm{M}+\mathrm{H}]^{+}$formed by electrospray ionization in positive mode (ESI-(+)) consistently lose the ascarylose moiety upon CID, corresponding to the neutral loss of 130.1 $\left[\mathrm{C}_{6} \mathrm{H}_{10} \mathrm{O}_{3}\right]$ (Fig. 2B). However, the large diversity of additional adducts formed during ESI-(+) including $\left[\mathrm{M}+\mathrm{NH}_{4}\right]^{+},[\mathrm{M}+\mathrm{Na}]^{+}$, and $[\mathrm{M}+\mathrm{K}]^{+}$, the latter of which are unsuitable precursors for MS/MS analysis, has so far precluded the exploitation of this fragmentation pathway for selective and sensitive ascaroside profiling. While HPLCESI-(-)-MS/MS precursor ion screening has proven extremely powerful in detecting novel ascarosides for subsequent chromatographic isolation and identification by NMR spectroscopy, ${ }^{[25]}$ resulting in the identification of more than 150 components from wild type and mutant $C$. elegans, ${ }^{[24]}$ it ultimately requires a triple quadrupole instrument, which is not widely available in many research laboratories. Consequently, we have recently developed a complementary GC-EIMS screen that facilitates the detection of basic ascarosides in trimethylsilyl (TMS)-derivatized crude nematode exometabolome extracts.[26,27] Systematic analysis of electron ionization (EI) mass spectra of ascaroside standards revealed highly characteristic marker signals corresponding to conserved fragment ions (Fig. 2C) such as $\mathrm{K} 1$ at $m / z$ 130.1 $\left[\mathrm{C}_{6} \mathrm{H}_{14} \mathrm{OSi}\right]^{\circ+}$, $\mathrm{A} 1$ at $m / z, 275.2\left[\mathrm{C}_{12} \mathrm{H}_{27} \mathrm{O}_{3} \mathrm{Si}_{2}\right]^{+}$, and $\mathrm{A} 2$ at $\mathrm{m} / \mathrm{z} \quad 185.1 \quad\left[\mathrm{C}_{9} \mathrm{H}_{17} \mathrm{O}_{2} \mathrm{Si}^{+}\right.$. In addition, we characterized side chain specific fragment ions such as $\mathrm{J} 1$ at $[\mathrm{M}-173]$ and $\mathrm{J} 2$ at $[\mathrm{M}$ 291] that facilitate the identification of compound-specific aglycones. Screening for the highly abundant, ascaroside-specific fragment ion at $\mathrm{m} / \mathrm{z}, 130.1$ highlights all basic ascaroside structures, which enabled the assignment of 200 ascaroside components in $C$. elegans wild type and mutant metabolomes, including isomeric compounds such as $(\omega-1)$ and $(\omega)$-linked, as well as $\alpha$-methyl branched isomers with side chains ranging from 3 to 33 carbons. ${ }^{[25]}$ Furthermore, GC-MS-based ascaroside screening also reveals yet unidentified ascaroside derivatives, whereas EI-induced fragmentation provides valuable information that could even facilitate structure assignment of unknown compounds.[27] However, GC-MS screening is unsuitable for highly complex ascaroside assemblies due to their limited volatility. ${ }^{[26,27]}$ In addition to these targeted screens, mass spectrometry has been employed for untargeted comparative analysis of $C$. elegans wild type and $d a f-22$ exometabolomes, followed by bioinformatic analysis and subsequent high resolution MS/MS based characterization, which resulted in the identification of a wealth of novel ascaroside derivatives. ${ }^{[28]}$

\section{The Co-option of Primary Metabolic Pathways in Ascaroside Biosynthesis}

The structural diversity of the ascarosides originates from the co-option of a primary metabolic pathway involved in peroxisomal $\beta$-oxidation of fatty acids to generate the various homologous aglycones, ${ }^{[29]}$ along with subsequent modifications, including the attachment of diverse building blocks to generate a complex modular library. Comparative analysis of C. elegans wild type and mutant metabolomes using MS/MS precursor ion screening facilitated the elucidation of ascaroside biosynthesis and the functional characterization of the corresponding genes 
<smiles>C1=CCCCC1</smiles><smiles>C[C@H](CCCCC=CC(=O)[O-])OC1O[C@H](C)C(O)C[C@H]1O</smiles><smiles>C1CCCCC1</smiles><smiles>CC1OC([O])C(O)C[C@H]1O</smiles>
$\mathrm{m} / \mathrm{z} 147.1$

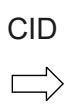<smiles>C1CCCOCCC1</smiles>

$\mathrm{m} / \mathrm{z} 73.1$<smiles>[Y16]O[C@@H]1C[C@H](O)[C@@H](C)O[C@@H]1OC(C)CC([Z])CC/C=C/C(=O)O</smiles><smiles>C[C@H](O)CCCC/C=C/[C@@H]([O])O</smiles>

C

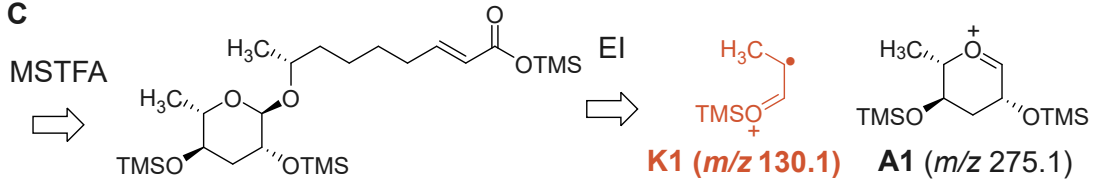<smiles>COC=[O+][13CH]C(C)CCCC/C=C/C(=O)OC</smiles><smiles>C[C+]CCCCC=CC(=O)OC</smiles>
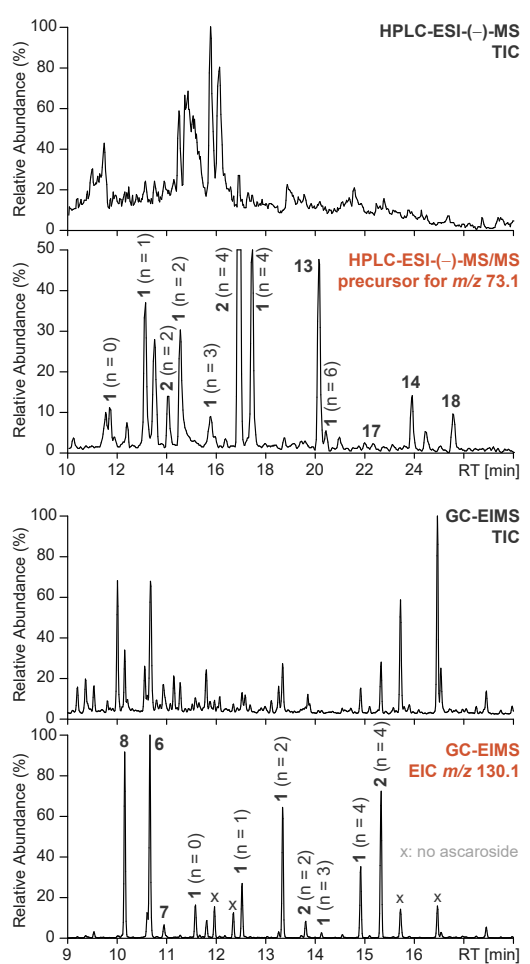

Fig. 2. Mass spectrometric fragmentations of ascarosides upon electrospray ionization (A, B) and electron ionization (C) generate characteristic fragments (red) that could be employed as marker for ascaroside selective screens.

(Fig. 3) including the acyl-S-CoA oxidase acox-1.1, enoyl-S-CoA hydratase maoc-1, (3R)-hydroxyacyl-S-CoA dehydrogenase $d h s-28$, and the 3-ketoacyl-S-CoA thiolase daf-22.[24] C. elegans and Pristionchus pacificus mutants showing defects in peroxisomal $\beta$-oxidation lack the short chain ascarosides characteristic for wild type metabolomes, and instead accumulate a diversity of long and very long chain precursors (with up to 33 carbons) ${ }^{[24,26]}$ including a series of very long chain methylketones and ethanolamides that represent shunt metabolites. ${ }^{[30,31]}$ Very long chain alkyland 2-hydroxyalkyl ascarosides such as 9 with side chains up to 35 carbons have previously been characterized in eggs of parasitic Ascaris species. ${ }^{[32]}$ These compounds are part of a lipid membrane that is believed to result in the extraordinary resilience of many nematode eggs against environmental factors. ${ }^{[33]}$ Very long chain 2-hydroxyalkyl ascarosides have also been reported from C. elegans ${ }^{[34]}$ and shown to be produced independently of peroxisomal $\beta$-oxidation, ${ }^{[26]}$ but their biosynthesis and relationship with the $\beta$-oxidation derived ascaroside signals is not fully understood. LC-MS/MS analysis of C. elegans mutants with defects in various acyl-S-CoA oxidase (acox) homologs along with in vitro assays with the heterologously expressed enzymes demonstrated considerable side chain preferences. ${ }^{[35]}$ For example, while the ACOX-1.1 homodimer processes both fatty acyl-S-CoA and ascaroside- $S$-CoA esters with medium length side chains, the ACOX-1.1-ACOX-1.3 heterodimer specifically processes ascarosides with a seven carbon side chain. ${ }^{[35]}$ Similarly, production of the synergistically active asc- $\omega \mathrm{C} 3(\mathbf{8})$ by chain shortening of its precursor is catalyzed by a dedicated ACOX-1.2 homodimer, the expression of which is upregulated in the presence of food. ${ }^{[35]}$ Ascaroside biosynthesis in Pristionchus pacificus under well fed conditions proceeds predominantly via Ppa-daf-22.1, whereas starvation induces expression of Ppa-daf-22.2 resulting in the production of a characteristic subset of ascaroside components. ${ }^{[31]}$ Gender-specific expression of acox-1.1 has been linked to the increased production of hermaphrodite attracting asc-C9 (1, $\mathrm{n}=4$, ascr\#10) in C. elegans males ${ }^{[36]}$ and similar gender-specific regulation of peroxisomal $\beta$-oxidation appears to modulate the production of sex pheromones such as $\mathbf{1 0}$ in the sour paste nematode Panagrellus redivivus. ${ }^{[37]}$ Taken together, these results suggest that the relative composition of basic ascarosides is at least in part regulated by differential expression of dedicated homologous genes that are part of the peroxisomal $\beta$-oxidation cycle.

\section{Ascaroside Signaling is Widely Conserved}

Basic ascarosides derived from the peroxisomal $\beta$-oxidation cycle are highly conserved in nematodes ${ }^{[27,38]}$ and modulate a large diversity of biological responses including dauer formation, ${ }^{118-20,39]}$ reproduction, ${ }^{[39,40]}$ stress resistance, ${ }^{[40,41]}$ lifespan, ${ }^{[41,42]}$ and behavior. ${ }^{[21,36]}$ Even small changes in molecular structures, ${ }^{[36,40,43]}$ synergistic effects, $[20-22,44]$ and variations in ascaroside compositions ${ }^{[40,45,46]}$ can dramatically alter their biological activity. Nematode responses towards ascarosides are highly strain specific, suggesting that ascaroside signaling might be dishonest ${ }^{[47]}$ and involved in inter-genotypic competition. ${ }^{[48,49]}$ In C. elegans, a synergistic blend of asc-C6-MK (6, $\mathrm{n}=2$; ascr\#2), ${ }^{[19]}$ asc$\Delta \mathrm{C} 9\left(2, \mathrm{n}=4\right.$; ascr\#3), ${ }^{[19]}$ and asc- $\omega \mathrm{C} 3$ $\left(\mathbf{8}\right.$, ascr\#5), ${ }^{[20]}$ acts as potent dauer-inducing signal. In pico- to attomolar amounts the predominantly male produced asc-C $9(\mathbf{1}, \mathrm{n}$ $=4$; ascr\#10) is a potent hermaphrodite attractant. ${ }^{[36]}$ Asc-C9 $(\mathbf{1}, \mathrm{n}=4$; ascr\#10) delays aging of the hermaphrodite germline thus postponing reproductive senescence. ${ }^{[40]}$ In Panagrellus redivivus the female produced asc-C7 (1, $\mathrm{n}=2$; ascr\#1) serves as a male attracting sex-pheromone. ${ }^{[37]}$ Similarly, in the trioecious nematode species Rhabditis sp. SB347 a sex pheromone blend consisting of asc-C5 (1, n = 0; ascr\#9) and asc-C7 (1, n $=2$; ascr\#1) is exclusively produced by females (and not by self-fertilizing hermaphrodites). Atto- to femtomolar amounts of asc-C5 and asc-C7 strongly attract males, thus increasing reproductive success of females and potentially stabilizing the occurrence of three genders. ${ }^{[50]}$ 


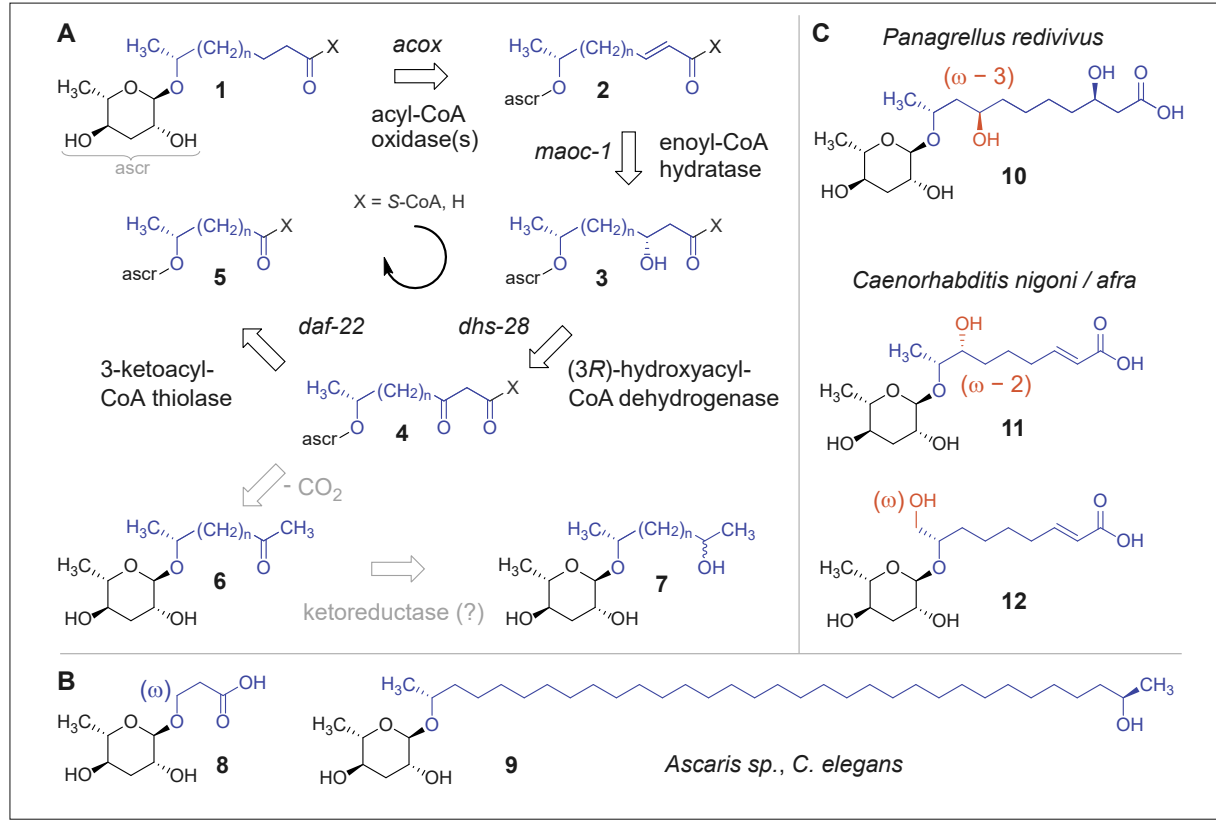

Fig. 3. Highly conserved ascaroside biogenesis via the peroxisomal $\beta$-oxidation cycle determines the length and functionality of the fatty acid derived aglycones $(A)$ and results in side chains ranging from 3 to 33 carbons (B). Resulting basic ascaroside structures can be further modified via species-specific $(\omega),(\omega-2)$, or $(\omega-3)$ hydroxylation $(C)$.

In addition, basic ascarosides are also involved in cross kingdom interactions, demonstrating their importance as key modulators in nematode chemical ecology. For example, infective juveniles of plant parasitic Meloidogyne sp. release a blend of simple ascarosides dominated by asc-C11 $\left(\mathbf{1}, \mathrm{n}=6\right.$; ascr\#18). ${ }^{[51]}$ Picomolar concentrations of asc-C11 induce plant defense responses and increase pathogen resistance, indicating that plants recognize ascarosides as a conserved molecular signature of nematodes. ${ }^{[51]}$ Similarly, a variety of simple ascarosides with side chains ranging from 5 to 9 carbons $(\mathbf{1}, \mathrm{n}=0,2,4)$ induce trap formation in nematophagous fungi including Arthrobotrys oligospora. ${ }^{[52]}$ In the pine wood nematode Bursaphelenchus xylophilus asc- $\Delta \mathrm{C6}(\mathbf{2}, \mathrm{n}=1)$, asc-C5 $(\mathbf{1}, \mathrm{n}=0)$, and asc-C9 $(\mathbf{1}, \mathrm{n}=4)$ coordinate nematode dispersal with the metamorphosis of its vector beetle Monochamus alternatus. ${ }^{[53]}$ Furthermore, basic ascarosides have been characterized in a large diversity of bacterivorous Caenorhabditis sp., ${ }^{[27,38]}$ as well as insect associated Pristionchus, Koernia, and Oscheius sp., entomopathogenic Heterorhabditis and Steinernema sp., and parasitic Pelodera strongyloides and Nippostrongyloides brasiliensis but their biological activities have remained largely unexplored.[38]

\section{Species-specific Modular Assembly of Primary Metabolic Building Blocks}

Downstream of the canonical $\beta$-oxidation cycle the basic ascarosides can undergo various species-specific modifications, thus giving rise to a large diversity of unique signaling molecules that represent the chemical language of nematodes. One specific signals. of the most basic species-specific modification is represented by tightly controlled $(\omega)$ and $(\omega-1)$, or $(\omega-3)$ hydroxylation of ascaroside aglycones (Fig. 3C). Analysis of the sour-paste nematode Panagrellus redivivus using traditional activity guided fractionation and MS/MS precursor ion screening revealed an exclusively maleproduced dihydroxylated ascaroside, asc8OH- $3 \mathrm{OH}-\mathrm{C} 11$ (10; dhas\#18), which serves as a potent female attractant, demonstrating gender-specific regulation of ascaroside biosynthesis via $(\omega-3)$-hydroxylation. ${ }^{[37]}$ Comparative GC-MS-based ascaroside profiling of 13 Caenorhabditis species further revealed two identical $(\omega-$ $2)$ and $(\omega)$-hydroxylated ascarosides, asc$7 \mathrm{OH}-\Delta \mathrm{C} 9$ (11) and asc-9-OH- $\Delta \mathrm{C} 9$ (12), in distantly related Caenorhabditis nigoni and Caenorhabditis afra, thus suggesting a polyphyletic origin of a presumed cytochrome P-450 mediated hydroxylation mechanism. ${ }^{[27]}$

In addition, the ascaroside core structures originating from peroxisomal $\beta$-oxidation serve as scaffolds for the highly species-specific attachment of additional building blocks originating from diverse primary metabolic pathways (Fig. 4), including fatty acid, citric acid, amino acid, carbohydrate, and nucleoside metab-

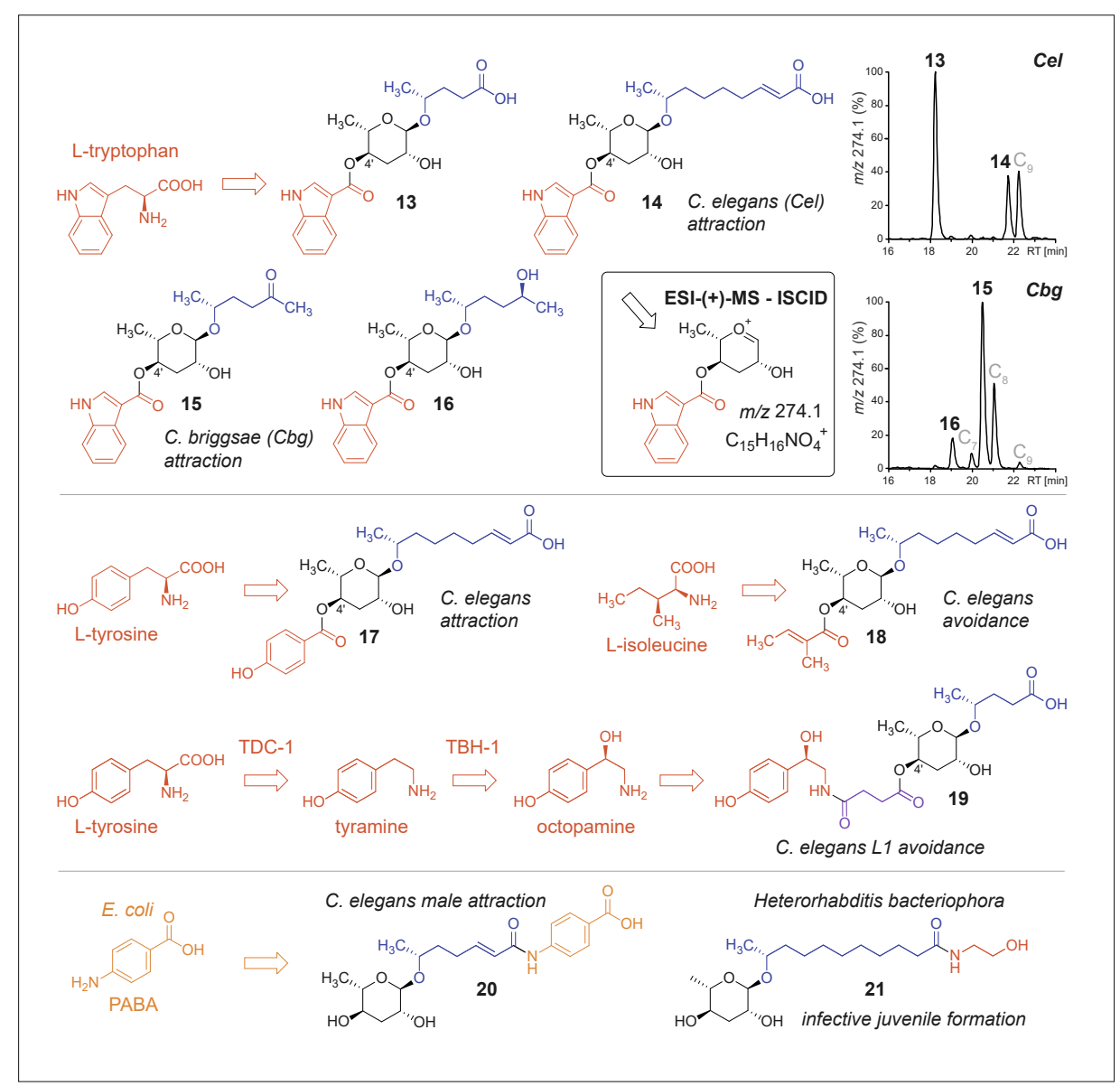

Fig. 4. Modification of ascaroside core structures downstream of the $\beta$-oxidation cycle via attachment of additional building blocks derived from primary metabolic pathways to generate species- 
olism, to generate highly complex modular assemblies. The indole ascarosides such as IC-asc-C5 (13, icas\#9) incorporate an indole-3-carboxylate unit at the 4'-position of the ascarylose and were first described as potent dauer pheromones with a bellshaped dose-response curve. ${ }^{[54]}$ Additional research demonstrated that IC-asc-C5 (13, icas\#9) and especially IC-asc- $\Delta$ C9 (14, icas\#3) elicit hermaphrodite attraction and aggregation in picomolar concentrations $\left(10^{-12} \mathrm{M}\right)$, several orders of magnitude lower than those required for dauer formation. ${ }^{23]}$ Comparative analysis of various Caenorhabditis species utilizing an indole ascaroside-specific HPLC-ESI-(+)-MS screen, based on a characteristic marker ion at $m / z, 274.1\left[\mathrm{C}_{15} \mathrm{H}_{16} \mathrm{NO}_{4}\right]^{+}$derived from in-source fragmentation (ISCID), revealed a high degree of species-specificity (Fig. 4). ${ }^{[4]}$ A synergistic blend of two hermaphrodite-produced indole ascarosides, ICasc-C6-MK (15, icas\#2) and IC-asc-C6$\mathrm{OH}$ (16, icas\#6.2) in femtomolar amounts $\left(10^{-15} \mathrm{~mol}\right)$ attracts $C$. briggsae males, suggesting a function as sex pheromone. ${ }^{[44]}$ Indole ascaroside production and response exhibits species and gender specificity, suggesting that the Caenorhabditis might employ these modifications to generate species-specific signals for intraspecies communication. ${ }^{[44]}$ Incorporation of deuterium labelled L-[D 5 -tryptophan into indole ascarosides in C. elegans confirmed the implication of amino acid metabolism in their biogenesis, ${ }^{[23]}$ whereas the conversion of exogenous asc- $\Delta \mathrm{C} 9(\mathbf{2}, \mathrm{n}$ $=4$; ascr\#3) into the corresponding indole ascaroside IC-asc- $\Delta$ C9 (14; icas\#3) in daf-22 mutant worms, which lack the ability to produce any short chain components, demonstrates that incorporation of the indole moiety occurs downstream of $\beta$-oxidation. ${ }^{[24]}$ Attachment of the indole-3-carboxylate unit (or other building blocks) is tightly controlled and highly side chain specific.[24,55] Production of 4'-acylated ascarosides with a five carbon side chain such as icas\#9 (13) depends on ACS-7, an acyl-S-CoA synthetase localized in gut granules, including lysosomerelated organelles (LROs) implicated with digestion of cellular waste and the biosynthesis of metabolites, thus, identifying a second hotspot for ascaroside biosynthesis. [55]

In addition to indole ascarosides, the 4-hydroxybenzoate group containing $\mathrm{HB}$ asc- $\Delta C 9$ (17, hbas\#3) of putative L-tyrosine origin represents one of the most potent attractants for $C$. elegans hermaphrodites, active in amounts down to 1 attomol $\left(10^{-18} \mathrm{~mol}\right),{ }^{[24]}$ whereas MB-asc- $\Delta \mathrm{C} 9(\mathbf{1 8}$, mbas\#3) with a 2-methyl-2E-butenoate (tiglate) moiety of presumed isoleucine origin $^{\text {[24] }}$ triggers strong avoidance behavior at $10 \mathrm{nM}$ even in the presence of food, ${ }^{[56]}$ demonstrating that modifications downstream of the $\beta$-oxidation cycle can completely inverse the biological activity. In line with their diverse biological functions the biogenesis of modular ascaroside signals strongly depends on the nematode's developmental stage and food availability. Upon starvation, first stage $C$. elegans larvae (L1) produce octopamine ascarosides such as OS-asc-C5 (19, osas\#9), which functions as an avoidance signal in micromolar concentrations but is much less active in the presence of food. The octopamine unit originates from L-tyrosine and its production depends on tyrosine decarboxylase $(t d c-1)$ and tyramine $\beta$-hydroxylase (tbh-1), thus linking neurotransmitter metabolism and ascaroside signaling. ${ }^{57]}$

Furthermore, ascarosides could also be attached to other building blocks originating from primary and secondary metabolic pathways via their side chain acyl moieties. Comparative analysis of wild type and daf-22 mutant metabolomes using differential analysis of 2D NMR spectra resulted in the identification of asc- $\Delta \mathrm{C} 7$ PABA (20, ascr\#8) as the first modular ascaroside component, which incorporates a para-aminobenzoic acid (PABA) moiety of presumed bacterial origin and constitutes a highly potent, synergistically active male attractant. ${ }^{22]}$ The entomopathogenic Heterorhabditis bacteriophora, which maintains a symbiotic relationship with Photorhabdus luminescens bacteria in order to infect and kill insects, produces a homologous series of $(\omega-1)$-linked ascarosides with side chains ranging from $\mathrm{C}_{5}$ to $\mathrm{C}_{17}$. Medium chain components with $\mathrm{C}_{11}^{5}$ are particularly prominent and the corre- sponding ethanolamide asc-C11-EA (21) has been identified as an inducer for the development of infective juveniles, a specialized larval stage similar to dauer larva that is capable of leaving the insect carcass and infecting new hosts.[58]

In addition, several modular ascarosides integrating additional carbohydrate units as well as glycoside and nucleoside building blocks have recently been identified in $C$. elegans (Fig. 5). A homologous series of highly polar $1 "-O$-acyl $\beta$-glucosides including asc- $\Delta$ C $9-\beta$ Glu (22, glas\#3) are retained in worm bodies and not excreted into the media. ${ }^{[24]}$ Ascaroside $O$-glucoside esters are strongly upregulated in the glo- 1 mutant, which lacks lysosome related organelles (LROs) implicated with ascaroside modification downstream of the $\beta$-oxidation cycle, ${ }^{[55]}$ possibly suggesting a role in ascaroside trafficking from the peroxisome to the LRO. Furthermore, various ascaroside $6 "-O$-acyl $\beta$-glucosides carrying anthranilate (25) or indole (26) aglycones have been detected using comparative MS analysis of $C$. elegans wild type and daf-22 mutant metabolomes. [28] The corresponding 1 "-O-glucosyl anthranilate (23) of unknown function has previously been shown to accumulate in LROs until it is finally released upon $C$. elegans' death. ${ }^{[59]}$ Production of indole$N$-glucoside (24) is part of C. elegans' detoxification mechanism ${ }^{[60]}$ and highly conserved in diverse Caenorhabditis species. Millimolar concentrations of indole, a quorum sensing signal of the E. coli food source, are lethal to $C$. elegans but rapidly metabolized to the non-toxic $N$-glucoside (24). [60] Subsequent attachment of $\mathbf{2 4}$ to

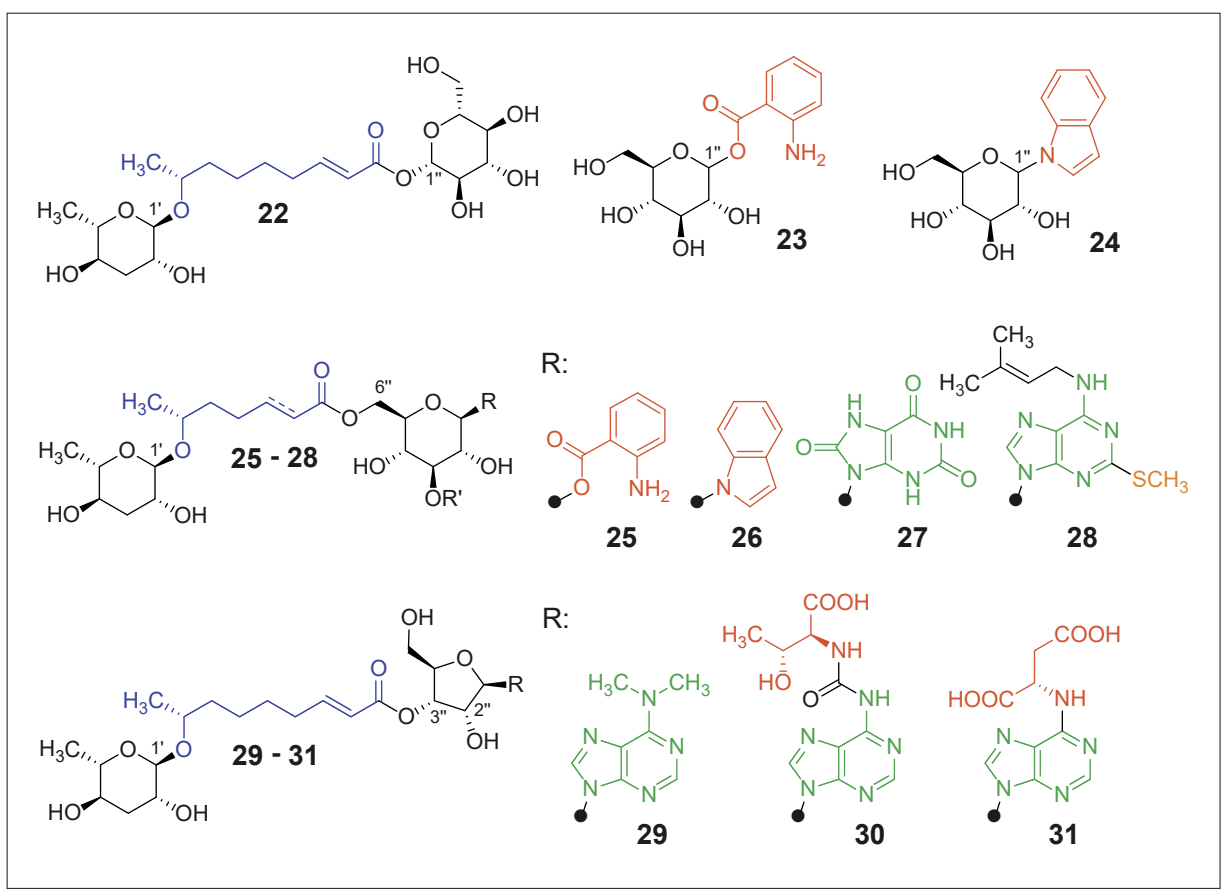

Fig. 5. Modular ascarosides from C. elegans integrating additional carbohydrate units as well as glycoside and nucleoside building blocks. 
basic ascarosides in order to produce $\mathbf{2 6}$ of yet unknown function links bacterial quorum sensing via a nematode detoxification pathway with ascaroside signaling. [28] Other glycoside-containing modular ascarosides integrate building blocks from nucleoside metabolism such as uric acid (27) or a methylthio derivative of the cytokinin isopentenyladenine $\left(\mathrm{i}^{6} \mathrm{~A}\right)(\mathbf{2 8})$. Additional $2 "-, 3$ "- $O$-isomeric riboside derivatives integrate a $N^{6}, N^{6}$-dimethyladenine unit (29) known as part of a conserved hairpin loop in small subunit ribosomal RNAs (rRNAs), a $N^{6}$-threonylcarbamoyl adenosine unit (30) known as ubiquitous modified nucleotide $\left(t^{6} \mathrm{~A}\right)$ in transfer RNAs (tRNAs) that decode codons starting with adenosine, as well as a succinyladenosine unit (31) known as an intermediate in the conversion of inosine monophosphate to adenosine monophosphate. ${ }^{[28]}$

Highly complex modular ascaroside assemblies are not restricted to C. elegans and have also been characterized in the necromenic Pristionchus pacificus that is associated with scarab beetles (Fig. 6). [61] While the general building blocks utilized by $C$. elegans and P. pacificus are somewhat similar, the resulting modular assemblies are highly species-specific. Most remarkably $P$. pacificus also produces an $\alpha$-glycolipid that is based on 2'-epi-ascarylose (L-paratose) linked via the $(\omega-1)$ position to a pentanoic acid aglycone, but it is unknown if part\#9 (32) is derived from longer chain paratoside precursors (similar to ascaroside biosynthesis) or if 2'-epimerization of the corresponding ascaroside occurs downstream of the $\beta$-oxidation cycle. ${ }^{[61]}$ Furthermore, part\#9 (32) serves as building block for several modular derivatives that integrate a $N^{6}$-threonylcarbamoyl adenosine moiety (33) from tRNA metabolism, which, however, carries an additional xylose unit instead of the canonical ribose. In micromolar concentrations part\#9 (32) and npar\#1 (33) modulate dauer formation, which is of central importance for the necromenic lifestyle.[61] The ureidoisobutyrate-containing ascarosides such as the dimeric ubas\#1 (34) are characterized by the thymine-derived building block at the 4'-position along with highly variable substitutions at the 2'-position of the ascarylose. ${ }^{[61]}$ Excretion and dauer formation response of various ascaroside signals including ubas\#1 (34) is highly strain specific, indicating a potential function in inter-genotypic competition. ${ }^{[49]}$ In addition, $P$. pacificus produces several ascaroside derivatives integrating a succinylated $\beta$-hydroxyphenylethylamine group such as pasc\#9 (35) and its anthranilic acid derivative, the penta-modular pasa\#9 (36). ${ }^{62]}$ Developmental plasticity in $P$. pacificus also includes mouthform dimorphism that is regulated by ascarosides. The homodi-

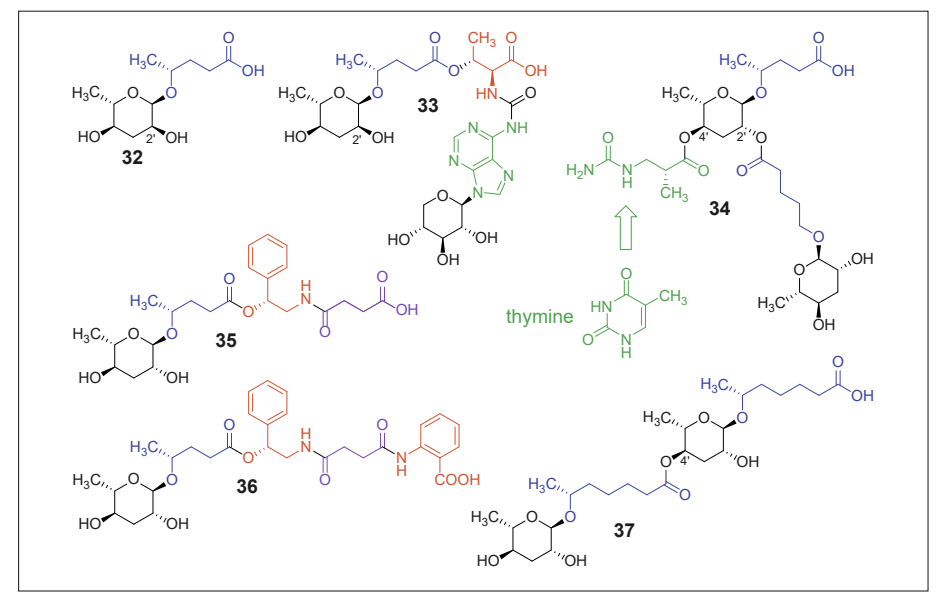

Fig. 6. Modular ascarosides from $P$. pacificus integrating building blocks from diverse primary metabolic pathways.

meric ascaroside dasc\#1 (37) triggers the development of the eurystomatous mouth form that enables $P$. pacificus to adapt a predatory lifestyle and feed on other nematodes. ${ }^{[61]}$ Although many more ascaroside structures have been detected and the number of identified compounds is continuously increasing, these examples should serve to demonstrate how nematodes utilize the combinatorial assembly of building blocks from diverse primary metabolic pathways to generate a species-specific chemical language.

\section{Conclusion \& Outlook}

While the molecular basis for nematode interactions and the chemical factors controlling developmental plasticity and behavior remained elusive for many decades, the recent development of novel analytical tools has facilitated the characterization of a complex modular glycolipid library and revolutionized our understanding of nematode chemical communication. In fact, using targeted ESI-MS/MS and GC-MS screens it is now much faster to comprehensively profile potential nematode signaling molecules, than it is to actually characterize their biological function. The large number of complex homologous structures makes their isolation or comprehensive total synthesis quite challenging. Consequently, while most of the newly discovered components are far from being fully characterized with respect to their biological activities, several new behavioral phenotypes have already been discovered using ascaroside as probes.

Our highly sensitive mass spectrometric screens facilitate the comprehensive ascaroside profiling in crude nematode exometabolomes and thereby enable us to eavesdrop on nematode chemical communication. Using these tools we are currently studying the diversity and complexity of the ascaroside language of nematodes. We follow a phylometabolomic approach and analyze a (continuously expanded) core collection of Caenorhabditis species that are closely related to the model organism $C$. elegans, as well as a wide diversity of unrelated species for comparative analysis. In addition to the identification of novel highly species-specific modular components and their functional characterization, we are equally interested to study the ecological significance of some widely conserved basic ascaroside signals, including their potential function in interspecies interactions between sympatric organisms, which could in theory range from competitive to cooperative and mutually beneficial interactions. Furthermore, we are exploring the various environmental and physiological factors that modulate ascaroside production, and study ascaroside biosynthetic pathways upstream and downstream of the canonical $\beta$-oxidation cycle, aiming to decipher the mechanisms that integrate diverse primary metabolic pathways and enable the broadcasting of the metabolic state of the organism within a population. While these studies have revealed a large diversity of yet undescribed modular ascarosides along with an even greater diversity of yet unidentified derivatives, we also found some nematode species that appear to produce only a very limited number of basic ascarosides, such as Oscheius tipulae ${ }^{[63]}$ or Rhabditis sp. SB347, [50] possibly suggesting that other yet unidentified and structurally unrelated classes of compounds might serve as additional signals in these organisms. In fact, it has been demonstrated that $C$. elegans also produces and responds to volatile cues, ${ }^{[64-66]}$ as well as some yet unidentified daf-22 independent components ${ }^{[67]}$ and hybrid PKS-NRPS peptides ${ }^{[68]}$ to modulate its behavior and development. Furthermore, our ongoing research also revealed that nematodes produce a large diversity of additional compounds by the combinatorial assembly of building blocks from primary and secondary metabolic pathways. The biological function and ecological significance of these species-specific nematodederived modular metabolites (NDMMs) is 
not understood. Considering that the various building blocks are highly conserved throughout the animal kingdom, it appears possible that similar strategies to create complex modular structures through combinatorial assembly of primary metabolites are not only prevalent among nematodes, but present in metazoans in general.

\section{Acknowledgements}

Financial support by the University of Neuchâtel (UniNE), the Swiss National Science Foundation (SNSF), the Max Planck Society (MPG), and the Jena School of Microbial Communication (JSMC) is gratefully acknowledged. I am extremely thankful to my mentors and colleagues for their support and to all my current and former co-workers for their dedication and hard work.

Received: March 2, 2018

[1] D. N. Greet, Nature 1964, 204, 96.

[2] a) C. D. Green, Helminthol. Abstracts, Ser. A 1980, 49, 327; b) C. D. Green, Helminthol. Abstracts, Ser. B 1980, 49, 81; c) L. W. Bone, H. H. Shorey, J. Chem. Ecol. 1978, 4, 595; d) L. W. Bone, Int. J. lnvertebr. Reprod. Dev. 1982, 5 , 311; e) R. N. Huettel, J. Nematol. 1986, 18, 3 e) B. M. MacKinnon, Parasitol. Today 1987, 3 , 156.

[3] a) S. H. von Reuss, F. C. Schroeder, Nat. Prod Rep. 2015, 32, 994; b) F. C. Schroeder, Chem Biol. 2015, 22, 7; c) R. A. Butcher, Nat. Chem Biol. 2017, 13, 577; d) R. A. Butcher, Nat. Prod. Rep. 2017, 34, 472.

[4] D. A. Neher, Annu. Rev. Phytopathol. 2010, 48 , 371

[5] a) F. Zhang, M, Berg, K. Dierking, M. A. Félix, M. Shapira, B. S. Samuel, H. Schulenburg, Front. Microbiol. 2017, 8, 485; b) P. Dirksen, S. A. Marsh, I. Braker, N. Heitland, S. Wagner R. Nakad, S. Mader, C. Petersen, V. Kowallik, P. Rosenstiel, M. A. Félix, H. Schulenburg, BMC Biol. 2016, 14, 38.

[6] M. A. Hassan, T. H. Pham, H. Shi, J. Zheng, Acta Agric. Scand. B 2013, 63, 420.

[7] G. Stepek, D. J. Buttle, I. R., Duce, J. M. Behnke, Int. J. Exp. Path. 2006, 87, 325.

[8] S. Brenner, Genetics 1974, 77, 71.

[9] R. E. Ellis, J. Y. Yuan and H. R. Horvitz, Annu. Rev. Cell Biol. 1991, 7, 663.

[10] A. Coulson, J. Sulston, S. Brenner and J. Karn, Proc. Natl. Acad. Sci. USA 1986, 83, 7821.

[11] The C. elegans Sequencing Consortium, Science 1998, 282, 2012.

[12] A. Fire, S. Q. Xu, M. K. Montgomery, S. A. Kostas, S. E. Driver, C. C. Mello, Nature 1998, $391,806$.

[13] M. Chalfie, Y. Tu, G. Euskirchen, W. W. Ward, D. C. Prasher, Science 1994, 263, 802.

[14] S. E. Hulme, G. M. Whitesides, Angew. Chem. Int. Ed. 2011, 50, 4774.

[15] J. W. Golden, D. L. Riddle, Science 1982, 218 , 578.

[16] J. W. Golden, D. L. Riddle, Mol. Gen. Genet. 1985, 198, 534

[17] P. Y. Jeong, M. Jung, Y. H. Yim, H. Kim, M. Park, E. Hong, W. Lee, Y. H. Kim, K. Kim, Y. K. Paik, Nature 2005, 433, 541

[18] Note on ascaroside nomenclature. In this review I will adhere to the structure-based nomencla-

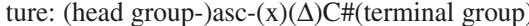
and the Small Molecule Identifiers (SMID, see: www.smid-db.org) in parallel whenever possible

[19] R. A. Butcher, M. Fujita, F. C. Schroeder, J. Clardy, Nature Chem. Biol. 2007, 3, 420.
[20] R. A. Butcher, J. R. Ragains, E. Kim, J. Clardy, Proc. Natl. Acad. Sci. USA 2008, 105, 14288.

[21] J. Srinivasan, F. Kaplan, R. Ajredini, C. Zachariah, H. T. Alborn, P. E. A. Teal, R. U. Malik, A. Edison, P. W. Sternberg, F. C. Schroeder, Nature 2008, 454, 1115.

[22] C. Pungaliya, J. Srinivasan, B. W. Fox, R. U. Malik, A. H. Ludewig, P. W. Sternberg, F. C. Schroeder, Proc. Natl. Acad. Sci. USA 2009, 106, 7708 .

[23] J. Srinivasan, S. H. von Reuss, N. Bose, A. Zaslaver, P. Mahanti, M. Ho, O. O'Doherty, A. S. Edison, P. W. Sternberg, F. C. Schroeder, PLoS Biol. 2012, 10, e1001237.

[24] S. H. von Reuss, N. Bose, J. Srinivasan, J. J. Yim, J. C. Judkins, P. W. Sternberg, F. C. Schroeder, J. Am. Chem. Soc. 2012, 134, 1817

[25] X. Zhang, J. H. Noguez, Y. Zhou, R. A. Butcher, 'Analysis of Ascarosides from Caenorhabditis elegans Using Mass Spectrometry and NMR Spectroscopy', in 'Pheromone Signaling: Methods and Protocols, Methods in Molecular Biology', Ed. K. Touhara, Vol. 1068, Springer Science+Business Media, LLC 2013, p 71-92.

[26] S. H. von Reuss, F. Dolke, C. Dong, Anal. Chem. 2017, 89, 10570

[27] C. Dong, D. K. Reilly, C. Bergame, F. Dolke, J. Srinivasan, S. H. von Reuss, J. Org. Chem. 2018, doi: 10.1021/acs.joc.8b00094.

[28] A. B. Artyukhin, Y. K. Zhang, A. E. Akagi, O. Panda, P. W. Sternberg, F. C. Schroeder, J. Am. Chem. Soc. 2018, doi: $10.1021 /$ jacs.7b11811.

[29] a) R. A. Butcher, J. R. Ragains, W. Li, G. Ruvkun, J. Clardy, H. Y. Mak, Proc. Natl. Acad. Sci. USA 2009, 106, 1875; b) H. J. Joo, Y. H. Yim, P. Y. Jeong, Y. X. Jin, J. E. Lee, H. Kim, S. K. Jeong, D. J. Chitwood, Y. K. Paik, Biochem. J. 2009, 422, 61; c) H. J. Joo, K. Y. Kim, Y. H. Yim, Y. X. Jin, H. Kim, M. Y. Kim, Y. K. Paik, J. Biol. Chem. 2010, 285, 29319.

[30] Y. Izrayelit, S. L. Robinette, N. Bose, S. von Reuss, A. S. Edison, F. C. Schroeder, ACS Chem. Biol. 2013, 8,314

[31] G. V. Markov, J. M. Meyer, O. Panda, A. B. Artyukhin, M. Claassen, H. Witte, F. C. Schroeder, R. J. Sommer, Mol. Biol. Evol. 2016, 33, 2506.

[32] J. P. Bartley, E. A. Bennett, P. A. Darben, J. Nat. Prod. 1996, 59, 921

[33] S. K. Olson, G. Greenan, A. B. Desai, T. MuellerReichert, K. F. Oegema, J. Cell. Biol. 2012, 198, 731.

[34] V. Zagoriy, V. Matyash, T. Kurzchalia, Chem. Biodivers. 2010, 7, 2016.

[35] X. Zhang, L. Feng, S. Chinta, P. Singh, Y. Wang, J. K. Nunnery, R. A. Butcher, Proc. Natl. Acad. Sci. USA 2015, 112, 3955 .

[36] Y. Izrayelit, J. Srinivasan, S. L. Campbell, Y. Jo, S. H. von Reuss, M. C. Genoff, P. W. Sternberg, F. C. Schroeder, ACS Chem. Biol. 2012, 7, 1321.

[37] A. Choe, T. Chuman, S. H. von Reuss, A. T. Dossey, J. Yim, R. Ajredini, A. A. Kolawa, F. Kaplan, H. T. Alborn, P. E. Teal, F. C. Schroeder, P. W. Sternberg, A. S. Edison, Proc. Natl. Acad. Sci. USA 2012, 109, 20949.

[38] A. Choe, S. H. von Reuss, D. Kogan, R. B. Gasser, E. G. Platzer, F. C. Schroeder, P. W. Sternberg, Curr. Biol. 2012, 22, 772.

[39] B. Wharam, L. Weldon, M. Viney, BMC Evol. Biol. 2017, 17, 197.

[40] E. Z. Aprison, I. Ruvinsky, PLoS Genet. 2015, 11, e1005729.

[41] H. A. Ludewig, Y. Izrayelit, D. Park, R. U. Malik, A. Zimmerman, P. Mahanti, B. W. Fox, A. Bethke, F. Doering, D. L. Riddle, F. C. Schroeder, Proc. Natl. Acad. Sci. USA 2013, 110 , 5522 .

[42] T. J. Maures, L. N. Booth, B. A. Benayoun, Y Izrayelit, F. C. Schroeder, A. Brunet, Science 2014, 343, 541

[43] K. A. Hollister, E. S. Conner, X. Zhang, M. Spell, G. M. Bernard, P. Patel, A. C. de Carvalho, R.
A. Butcher, J. R. Ragains, Bioorg. Med. Chem. 2013, 21, 5754

[44] C. Dong, F. Dolke, S. H. von Reuss, Org. Biomol. Chem. 2016, 14, 7217

[45] E. Z. Aprison, I. Ruvinsky, Curr. Biol. 2016, 26, 2827.

[46] E. Z. Aprison, I. Ruvinsky, Curr. Biol. 2017, 27 , 1.

[47] M. Viney, S. Harvey, PLoS Genet. 2017, 13, e1007046.

[48] S. A. Diaz, V. Brunet, G. C. Lloyd-Jones, W. Spinner, B. Wharam, M. Viney, BMC Evol. Biol. 2014, 14, 46 .

[49] N. Bose, J. M. Meyer, J. J. Yim, M. G. Mayer, G. V. Markov, A. Ogawa, F. C. Schroeder, R. J. Sommer, Curr. Biol. 2014, 24, 1536

[50] J. Chaudhuri, N. Bose, S. Adams, G. Zuco, S Tandonnet, T. Naia, V. Kache, M. Parihar, S. H. von Reuss, F. C. Schroeder, A. Pires-da Silva, Sci. Rep. 2015, 5, 17676.

[51] P. Manosalva, M. Manohar, S. H. von Reuss, S. Chen, A. Koch, F. Kaplan, A. Choe, R. J. Micikas, X. Wang, K. H. Kogel, P. W. Sternberg, V. M. Williamson, F. C. Schroeder, D. Klessig, D. F. Nat. Commun. 2015, 6, 7795.

[52] Y. P. Hsueh, P. Mahanti, F. C. Schroeder, P. W. Sternberg, Curr. Biol. 2013, 23, 83.

[53] L. Zhao, X. Zhang, Y. Wei, J. Zhou, W. Zhang, P. Qin, S. Chinta, X. Kong, Y. Liu, H. Yu, S. Hu, Z Zou, R. A. Butcher, J. Sun, Nat. Commun. 2016, 7,12341

[54] R. A. Butcher, J. R. Ragains, J. Clardy, Org. Lett. 2009, 11,3100 .

[55] O. Panda, A. E. Akagi, A. B. Artyukhin, J. C. Judkins, H. H. Le, P. Mahanti, S. M. Cohen, P. W. Sternberg, F. C. Schroeder, Angew. Chem. Int Ed. 2017, 56, 4729

[56] Y. K. Zhang, M. A. Sanchez-Ayala, P. W. Sternberg, J. Srinivasan, F. C. Schroeder, Org. Lett. 2017, 19, 2837.

[57] A. B. Artyukhin, J. J. Yim, J. Srinivasan, Y Izrayelit, N. Bose, S. H. von Reuss, Y. Jo, J. M. Jordan, L. R. Baugh, M. Cheong, P. W. Sternberg, L. Avery, F. C. Schroeder, J. Biol. Chem. 2013, 288, 18778.

[58] J. H. Noguez, E. S. Conner, Y. Zhou, T. A. Ciche, J. R. Ragains, R. A. Butcher, ACS Chem. Biol. 2012, 7, 961 .

[59] C. Coburn, E. Allman, P. Mahanti, A. Benedetto, F. Cabreiro, Z. Pincus, F. Matthijssens, C. Araiz, A. Mandel, M. Vlachos, S. A. Edwards, G Fischer, A. Davidson, R. E. Pryor, A. Stevens, F. J. Slack, N. Tavernarakis, B. P. Braeckman, F. C. Schroeder, K. Nehrke, D. Gems, PLoS Biol. 2013, 11, e1001613.

[60] G. S. Stupp, S. H. von Reuss, Y. Izrayelit, R. Ajredini, F. C. Schroeder, A. S. Edison, ACS Chem. Biol. 2013, 8, 309

[61] N. Bose, A. Ogawa, S. H. von Reuss, J. J. Yim, R. J. Sommer, F. C. Schroeder, Angew. Chem. Int. Ed. 2012, 51, 12438

[62] J. J. Yim, N. Bose, J. M. Meyer, R. J. Sommer, F. C. Schroeder, Org. Lett. 2015, 17, 1648.

[63] C. Kuhlisch, S. H. von Reuss, 2015, unpublished results.

[64] J. R. Chasnov, W. K. So, C. M. Chan, K. L. Chow, Proc. Natl. Acad. Sci. USA 2007, 104, 6730.

[65] D. H. W. Leighton, A. Choe, S. Y. Wu, P. W. Sternberg, Proc. Natl. Acad. Sci. USA 2014, 111, 17905.

[66] F. Borne, K. R. Kasimatis, P. C. Phillips, PLoS ONE 2017, 12, e0189679.

[67] Y. Zhou, M. Loeza-Cabrera, Z. Liu, B. AlemanMeza, J. K. Nguyen, S. K. Jung, Y. Choi, Q. Shou, R. A. Butcher, W. Zhong, Genetics 2017, $206,1469$.

[68] Q. Shou, L. Feng, Y. Long, J. Han, J. K. Nunnery, D. H. Powell, R. A. Butcher, Nat. Chem. Biol. 2016, 770. 